\title{
Decreased serum level of NGF in alcohol-dependent patients with declined executive function
}

This article was published in the following Dove Press journal:

Neuropsychiatric Disease and Treatment

13 November 2014

Number of times this article has been viewed

\author{
Hwallip Bae' \\ Youngsun $\mathrm{Ra}^{1}$ \\ Changwoo $\mathrm{Han}^{2}$ \\ Dai-Jin Kim ${ }^{3}$ \\ 'Department of Psychiatry, Myongji \\ Hospital, Goyang, ${ }^{2}$ Department \\ of Psychiatry, Keyo Hospital, \\ Uiwang, ${ }^{3}$ Department of Psychiatry, \\ Seoul St Mary's Hospital, College \\ of Medicine, Catholic University \\ of Korea, Seoul, South Korea
}

\begin{abstract}
The role of neurotrophic factors has been highlighted as a cause of decline in the cognitive function of alcohol-dependent patients. It is known that nerve-growth factor (NGF), one of the neurotrophins, is related to the growth and differentiation of nerve cells, as well as to a decline in cognitive function. The purpose of this study was to investigate the relationship between decreased NGF levels and cognitive decline in alcohol-dependent patients. The serum concentration of NGF was measured in 38 patients with chronic alcohol dependence, and several neuropsychological tests were also performed for cognitive function assessment. The results indicated a significant correlation between serum NGF level and the trail-making test part B, which evaluates executive function, but did not show a significant correlation with other cognitive function tests. An increased serum level of NGF was associated with a decreased completion time in the trail-making test $\mathrm{B}$, and this finding indicates that a high serum level of NGF is related to greater executive function. This finding may imply a protective role of NGF in preventing neuron damage among patients with alcohol dependence. Larger controlled studies will be necessary in the future to investigate this issue further.
\end{abstract}

Keywords: nerve-growth factor, alcohol dependence, executive function, trail-making test

\section{Introduction}

It has been well acknowledged that alcohol-dependent patients experience a decline in their cognitive function even after the cessation of alcohol consumption. ${ }^{1,2}$ Although learning ability and memory are recovered with time, abstract reasoning, problemsolving skill, perception, and motor function are lower than in a normal control group after 1 year of abstinence. ${ }^{3}$

The cognitive decline in alcohol-dependent patients that continues after abstinence is most likely due to neuronal damage caused by alcohol. ${ }^{4-6}$ Recently, the role of neurotrophic factors, such as brain-derived neurotrophic factor (BDNF) and nerve-growth factor (NGF), has received focus among several factors that are involved in the protecting mechanism of alcohol-induced neuronal damage. ${ }^{7}$

NGF is one of the neurotrophins involved in the growth and differentiation of nerve cells and prevention of damage to mature neurons. It may also play an important role in protecting from alcohol-induced neurotoxicity. ${ }^{8-11}$ There are several studies in alcohol-dependent patients with different findings on NGF. Heberlein et al suggested that NGF rises during acute alcohol intoxication and declines during the withdrawal period. ${ }^{12}$ Other studies reported that the NGF level drops in patients who have been abstinent for longer than 30 days when compared to a control group. In particular, those who had a family history of alcoholism showed a greater decrease. ${ }^{13}$ It seems that NGF increased in the intoxication period for the compensation of the neurotoxic effects of alcohol, but eventually decreased in chronic alcohol-dependent patients, 
even in the abstinence period. The number of studies is not sufficient yet for consistent results.

Among a handful of studies on the relationship between NGF and patients' cognitive function, Jockers-Scherübl et al reported that even though alcohol-dependent patients had increased levels of serum NGF and the levels were even higher with the symptoms of withdrawal delirium, the serum NGF level in patients with persistent amnesic disorder (Korsakoff's syndrome) showed no changes and was different from the control group. ${ }^{14}$

Furthermore, in experiments with animals, when rats were administered with NGF after they were withdrawn from chronic treatment with alcohol, their spatial learning recovered and the length density of hippocampal cholinergic fibers was restored. ${ }^{15}$

Recent studies have shown the value of NGF in investigating the pathogenesis and its potential as a biological marker in alcohol-dependent patients. ${ }^{13}$ Also, animal studies have demonstrated its potential use in long-term treatment. ${ }^{15}$ As mentioned earlier, however, there are few studies that have investigated the correlation between NGF and cognitive function. Therefore, the authors measured the serum NGF level in alcohol-dependent patients during the abstinence period and then conducted several neuropsychological tests as a cognitive function-evaluation tool in order to determine the correlation between them.

\section{Materials and methods Subjects}

The subjects in this study were 38 patients admitted to the Alcohol Treatment Center at a psychiatric institution in Gyeonggi Province, South Korea. The patients were diagnosed with alcohol dependence by two psychiatrists according to Diagnostic and Statistical Manual of Mental Disorders (DSM)-IV criteria. The age range was 38-61 years, and patients with psychiatric disorders other than depression were excluded from the study. Exclusion criteria also included other substance abuse, history of severe head injury, and neurological disorders. Patients in their detoxification period were not included either. Also, patients with withdrawal symptoms were excluded. Patients with a confirmed abstinence period of at least 1 week by admission in a closed ward were allowed to participate in the study.

None of the participants were on antidepressant or antipsychotic treatment, although most of them had been prescribed either antianxiolytics or sleeping pills at low doses, hepatotonics, and multivitamins. Patients were taking the anxiolytic lorazepam $1 \mathrm{mg}$ or less and the hypnotic zolpidem $10 \mathrm{mg}$ or less. Multivitamins were mainly vitamins $\mathrm{B}_{1}$ and $\mathrm{B}_{6}$. The ingredients of hepatotonics and the exact numbers of subjects who were taking these medications were unascertainable.

Demographic and clinical characteristics of the subjects are displayed in Table 1. All subjects were men. They agreed to participate in the study and signed an informed consent form after the researchers explained the study details. The study was approved by the Institutional Review Board of Seoul St Mary's Hospital, Seoul, South Korea.

\section{Clinical assessments}

A survey was used to collect the demographic variables, including subjects' age, education, age of first alcohol consumption, frequency of alcohol consumption, average amount of consumed alcohol, and abstinence periods of alcohol. Neuropsychological tests - verbal fluency, wordlist memory test, word-list recall, word-list recognition, constructional praxis, constructional recall, Boston naming test, Mini Mental Status Examination - Korean (MMSE-K), and trail-making test $\mathrm{A}$ and $\mathrm{B}$ - were used to evaluate cognitive function. These neuropsychological tests evaluate language, memory, constructional ability, and executive function. Two clinical psychologists conducted the tests on each patient.

Table I Correlations among demographics, alcohol-related data, NGF, and TMT

\begin{tabular}{|c|c|c|c|c|}
\hline & Mean \pm SD & TMT-A & TMT-B & NGF \\
\hline \multicolumn{5}{|l|}{ Demographic data } \\
\hline Age, years & $51.18 \pm 6.38$ & 0.253 & 0.173 & -0.157 \\
\hline \multicolumn{5}{|l|}{ Alcohol related data } \\
\hline Onset age of drinking & $21.15 \pm 7.98$ & $0.461 * *$ & 0.305 & 0.116 \\
\hline Frequency of alcohol consumed (per week) & $4.15 \pm 2.21$ & 0.114 & -0.263 & -0.122 \\
\hline Daily amount of alcohol consumed (standard drink) & $3.18 \pm 2.14$ & 0.024 & 0.066 & $-0.322^{*}$ \\
\hline First age of alcohol treatment & $38.52 \pm 8.22$ & 0.095 & 0.103 & 0.050 \\
\hline Total abstinence period, days & $185.66 \pm 474.35$ & -0.038 & -0.065 & -0.166 \\
\hline
\end{tabular}

Notes: $* P<0.05 ; * * P<0.01$.

Abbreviations: TMT, trail-making test; NGF, nerve-growth factor; SD, standard deviation. 


\section{Enzyme-linked immunosorbent assay}

The subjects' blood was drawn by a nurse between 10 and $11 \mathrm{am}$. Ten milliliters of blood was collected into an ethylenediaminetetraacetic acid-coated tube, immediately centrifuged until the serum was separated, and stored at $-80^{\circ} \mathrm{C}$ for further analysis.

NGFs were measured using Human Adipokine Magnetic Bead Panels 2 - Endocrine Multiplex Assay (HADK2MAG$61 \mathrm{~K})$, manufactured by Millipore (Seoul, South Korea). The test was conducted according to the standard guidelines suggested by the manufacturer. The concentration of the plates was corrected through the dilution factor of the standard curve.

\section{Statistical analysis}

A Pearson correlation analysis was used to determine the correlation among the demographic variables, the result of neuropsychological tests, and NGF. Student's $t$-test was used to determine whether there was a difference in the serum level of NGF or the results of neuropsychological tests between patients with a positive and negative family history. Likewise, the $t$-test was used for comparison between patients who were positive and negative for withdrawal-symptom history. Analysis of variance (ANOVA) was conducted to determine if there was a difference in the serum level of NGF or the results of neuropsychological test by education level (junior high, high school, professional college, 4-year university). Windows SPSS version 16.0 was used as the software tool for statistical analysis, and the significance level was set at $P<0.05$.

\section{Results}

\section{Enzyme-linked immunosorbent assay}

The mean serum level of NGF and standard deviation was $26.74 \pm 10.08 \mathrm{pg} / \mathrm{mL}$.

\section{Demographic characteristics and alcohol-related data}

There were 30 patients with a family history of alcoholism among the 38 patients. The number of patients with a history of alcohol-withdrawal symptoms was 32 . The $t$-test revealed no significant difference in the serum level of NGF or the results of neuropsychological testing according to family history or alcohol-withdrawal symptom history.

The average duration of education was approximately 10 years. ANOVA showed no significant difference in the results from neuropsychological tests or the level of NGF by education level. The results of the Pearson correlation test of age and other alcohol-related data are presented in Table 1.
No significant correlation with either neuropsychological test results or NGF level was shown for age. However, in alcohol-related data, the onset age of drinking had a positive correlation with trail-making test $\mathrm{A}$, and the amount of alcohol consumed daily had a negative correlation with NGF serum level (Table 1).

\section{Association between NGF and neuropsychological test in patients with alcohol dependence}

Table 2 shows the association between NGF and neuropsychological test results in alcohol-dependent patients. The results revealed no significant correlation between levels of NGF and the neuropsychological test results, except the trail-making test $\mathrm{B}$. The correlation between NGF and the trail-making test $\mathrm{B}$ was significant ( $r=-0.324, P=0.046$ ).

\section{Correlation between NGF and trail-making test indices}

Associations between NGF and derived trail-making test indices B - A, B/A, and B - A/A were examined. Significant correlations between NGF level and derived trail-making test indices $\mathrm{B}-\mathrm{A}, \mathrm{B} / \mathrm{A}$, and $\mathrm{B}-\mathrm{A} / \mathrm{A}$ were also revealed from the investigation (Table 3).

\section{Discussion}

The purpose of this study was to investigate the relationship between the level of serum NGF and the decline in cognitive function of alcohol-dependent patients during the abstinence period. Among several cognitive function tests, the trail-making test $B$ and NGF level demonstrated significant correlation. The increased NGF was associated with a lower trail-making test B score, indicating faster performance speed.

Table 2 Correlations between NGF and neuropsychological tests for cognitive function evaluation

\begin{tabular}{lll}
\hline Neuropsychological tests & Mean \pm SD & NGF $\boldsymbol{r}$ \\
\hline Verbal fluency & $15.29 \pm 3.67$ & 0.173 \\
Word-list memory & $18.13 \pm 4.03$ & $0.04 \mathrm{I}$ \\
Word-list recall & $6.45 \pm 2.13$ & -0.070 \\
Word-list recognition & $9.34 \pm 0.94$ & -0.038 \\
Constructional praxis & $10.42 \pm \mathrm{I} .18$ & 0.050 \\
Constructional recall & $8.66 \pm 2.53$ & $-0.01 \mathrm{I}$ \\
Boston naming test & $12.18 \pm \mathrm{I} .92$ & -0.084 \\
MMSE-K & $26.84 \pm 3.1 \mathrm{I}$ & 0.134 \\
Trail making test A & $50.87 \pm 24.57$ & $-0.10 \mathrm{I}$ \\
Trail making test B & $190.74 \pm 91.33$ & $-0.324 *$ \\
\hline
\end{tabular}

Note: $* P<0.05$

Abbreviations: NGF, nerve-growth factor; SD, standard deviation; MMSE-K, MiniMental State Examination - Korean. 
Table 3 Correlations between NGF- and TMT-derived indices

Variable TMT A TMT B TMT B-A TMT B/A TMT B - A/A

\begin{tabular}{llllll}
\hline NGF & -0.101 & $-0.326 *$ & $-0.35 I^{*}$ & $-0.358^{*}$ & $-0.358^{*}$
\end{tabular}

Note: $* p<0.05$.

Abbreviations: TMT, trail-making test; NGF, nerve-growth factor.

The trail-making test includes motor components as well as complex visual scanning, so it can be used to test visual motor tracking and such abilities as general attention and psychomotor speed. ${ }^{16,17}$ Since the trail-making test $B$ requires more complex mental activity than test $\mathrm{A}$, it is known to be more sensitive to brain damage. ${ }^{18,19}$

In addition, there have been attempts in several studies to locate brain lesions or identify impairment in specific brain functions based on the difference score $(\mathrm{A}-\mathrm{B})$, score ratio (A/B), and proportional score $(\mathrm{B}-\mathrm{A} / \mathrm{A})$. Derived indices especially indicated impairment in executive function..$^{20-22}$

In this study, all derived indices also displayed significant correlation with the NGF serum level. This finding indicates that executive functions of alcohol-dependent patients have significant correlations with NGF. ${ }^{18-20}$ In other words, the NGF level was higher in patients with higher executive function, which is in agreement with research findings that NGF prevents neuronal cell loss and atrophy in neurodegenerative disorders, such as Alzheimer's disease. ${ }^{7,23,24}$ It also agrees with research findings that NGF protects nerve cells from neurotoxicity due to alcohol..$^{6,8-11,15}$

In the study of Jockers-Scherübl et al the serum NGF level was higher in alcohol-dependent patients than in the control group..$^{14}$ In the present study, the daily amount of alcohol consumed showed a negative correlation with NGF levels (Table 1), which means that more alcohol intake led to lower levels of NGF. However, considering Jockers-Scherübl et al's qualified 8 days of withdrawal, and the fact that patients' mean abstinence period was more than 185 days in our study, we can speculate the NGF level rise during the early withdrawal period was for neuroprotection, but afterwards, especially for the cognitive dysfunctioning patients, low levels of serum NGF were observed. In the study of Jockers-Scherübl et al patients with amnesic disorder (Korsakoff's syndrome) had similar levels of serum NGF as the control group, which is in agreement with the results of the present study. ${ }^{14}$

Therefore, in addition to the confirmation of the neuroprotective effects of NGF in alcohol-dependent patients, its role as a stage marker in alcohol-related diseases mentioned by Jockers-Scherübl et al and its therapeutic effect shown in Lukoyanov et al's experiments with animals are valuable findings that should be further investigated. ${ }^{15}$
The daily amount of consumed alcohol had a significant negative correlation with the NGF level among the demographic data related to alcohol (Table 1). This finding indicates that in general, the greater the amount of alcohol consumed per day the lower the level of NGF. Also, the daily amount of consumed alcohol had a greater influence on the NGF level than the duration of alcohol dependence, abstinence period, family history, or withdrawal symptoms.

Limitations of this study were a lack of comparison between alcohol-dependent patients and the control group, a relatively small study population, and a subject group comprised entirely of males. Also, an investigation of smoking history was not conducted. There are previous studies on nicotine's effect on cognitive function or level of NGF. ${ }^{25-27}$ Therefore, the possibility that nicotine use affects executive function or serum levels of NGF in alcoholdependent patients exists.

Although they took neither antidepressants nor antipsychotic medications, most of the patients were taking multivitamins, hepatotonics, and low dose of anxiolytics or sleeping pills. The negative effect of benzodiazepine on cognitive function and positive effects of vitamin A, D, or B complex on cognition are well known. ${ }^{28-30}$ However, the influences of these medications were not considered. As far as we know, the effect of hepatotonics on cognitive function is unknown, and these drugs' effect on NGF is not known either, except a few studies of vitamin $A$ or $B_{12}$ 's effect on NGF. ${ }^{31,32}$

In addition to this, it is difficult to know from this study how long the NGF serum level increase persists during the abstinence period, because a check of NGF levels was done only once in this study. Therefore, this is considered one of the limitations of this study. Future studies should take these points into consideration. We recommend further studies investigate the therapeutic effect of NGF in patients with declined cognitive function.

\section{Acknowledgment}

The study was supported by a grant from the Korean Health Technology R\&D Project, Ministry of Health and Welfare, South Korea (HI11C13320000).

\section{Disclosure}

The authors report no conflicts of interest in this work.

\section{References}

1. Eckardt MJ, Stapleton JM, Rawlings RR, Davis EZ, Grodin DM. Neuropsychological functioning in detoxified alcoholics between 18 and 35 years of age. Am J Psychiatry. 1995;152(1):53-59.

2. Kopera M, Wojnar M, Brower K, et al. Cognitive functions in abstinent alcohol-dependent patients. Alcohol. 2012;46(7):665-671. 
3. Fein G, Bachman L, Fisher S, Davenport L. Cognitive impairments in abstinent alcoholics. West J Med. 1990;152(5):531-537.

4. Tateno M, Saito T. Biological studies on alcohol-induced neuronal damage. Psychiatry Investig. 2008;5(1):21-27.

5. Miller MW. Limited ethanol exposure selectively alters the proliferation of precursor cells in the cerebral cortex. Alcohol Clin Exp Res. 1996; 20(1):139-143.

6. Nixon K, Crews FT. Binge ethanol exposure decreases neurogenesis in adult rat hippocampus. $J$ Neurochem. 2002;83(5):1087-1093.

7. Connor B, Dragunow M. The role of neuronal growth factors in neurodegenerative disorders of the human brain. Brain Res Brain Res Rev. 1998;27(1):1-39.

8. Seabold GK, Luo J, Miller MW. Effect of ethanol on neurotrophinmediated cell survival and receptor expression in cultures of cortical neurons. Brain Res Dev Brain Res. 1998;108(1-2):139-145.

9. Heaton MB, Mitchell JJ, Paiva M. Overexpression of NGF ameliorates ethanol neurotoxicity in the developing cerebellum. $J$ Neurobiol. 2000;45(2):95-104.

10. Moore DB, Madorsky I, Paiva M, Barrow Heaton M. Ethanol exposure alters neurotrophin receptor expression in the rat central nervous system: effects of prenatal exposure. J Neurobiol. 2004;60(1):101-113.

11. Moore DB, Madorsky I, Paiva M, Barrow Heaton M. Ethanol exposure alters neurotrophin receptor expression in the rat central nervous system: effects of neonatal exposure. J Neurobiol. 2004;60(1):114-126.

12. Heberlein A, Bleich S, Bayerlein K, et al. NGF plasma levels increase due to alcohol intoxication and decrease during withdrawal. Psychoneuroendocrinology. 2008;33(7):999-1003.

13. Yoon SJ, Roh S, Lee H, et al. Possible role of nerve growth factor in the pathogenesis of alcohol dependence. Alcohol Clin Exp Res. 2006;30(6):1060-1065.

14. Jockers-Scherübl MC, Bauer A, Kuhn S, et al. Nerve growth factor in serum is a marker of the stage of alcohol disease. Neurosci Lett. 2007; 419(1):78-82.

15. Lukoyanov NV, Pereira PA, Paula-Barbosa MM, Cadete-Leite A. Nerve growth factor improves spatial learning and restores hippocampal cholinergic fibers in rats withdrawn from chronic treatment with ethanol. Exp Brain Res. 2003;148(1):88-94.

16. Crowe SF. The differential contribution of mental tracking, cognitive flexibility, visual search, and motor speed to performance on parts A and B of the Trail Making Test. J Clin Psychol. 1998;54(5):585-591.

17. Miner T, Ferraro FR. The role of speed of processing, inhibitory mechanisms, and presentation order in trail-making test performance. Brain Cogn. 1998;38(2):246-253.

18. Arbuthnott K, Frank J. Trail making test, part B as a measure of executive control: validation using a set-switching paradigm. J Clin Exp Neuropsychol. 2000;22(4):518-528.
19. Gaudino EA, Geisler MW, Squires NK. Construct validity in the Trail Making Test: what makes Part B harder? J Clin Exp Neuropsychol. 1995;17(4):529-535.

20. Sánchez-Cubillo I, Periáñez JA, Adrover-Roig D, et al. Construct validity of the Trail Making Test: role of task-switching, working memory, inhibition/interference control, and visuomotor abilities. J Int Neuropsychol Soc. 2009;15(3):438-450.

21. Periáñez JA, Ríos-Lago M, Rodríguez-Sánchez JM, et al. Trail Making Test in traumatic brain injury, schizophrenia, and normal ageing: sample comparisons and normative data. Arch Clin Neuropsychol. 2007;22(4):433-447.

22. Stuss DT, Bisschop SM, Alexander MP, Levine B, Katz D, Izukawa D. The Trail Making Test: a study in focal lesion patients. Psychol Assess. 2001;13(2):230-239.

23. Aloe L, Rocco ML, Bianchi P, Manni L. Nerve growth factor: from the early discoveries to the potential clinical use. $J$ Transl Med. 2012;10:239.

24. Schindowski K, Belarbi K, Buee L. Neurotrophic factors in Alzheimer's disease: role of axonal transport. Gene Brain Behav. 2008;7 Suppl 1: 43-56.

25. White HK, Levin ED. Four-week nicotine skin patch treatment effects on cognitive performance in Alzheimer's disease. Psychopharmacology. 1999;143(2):158-165

26. French KL, Granholm AC, Moore AB, Nelson ME, Bimonte-Nelson HA. Chronic nicotine improves working and reference memory performance and reduces hippocampal NGF in aged female rats. Behav Brain Res. 2006;169(2):256-262.

27. Hernandez CM, Terry AV Jr. Repeated nicotine exposure in rats: effects on memory function, cholinergic markers and nerve growth factor Neuroscience. 2005;130(4):997-1012.

28. Health Quality Ontario. Vitamin B12 and cognitive function: an evidence-based analysis. Ont Health Technol Assess Ser. 2013;13(23): $1-45$.

29. Lu'o'ng KV, Nguyen LT. The beneficial role of vitamin D in Alzheimer's disease. Am J Alzheimers Dis Other Demen. 2011;26(7):511-520.

30. Olson CR, Mello CV. Significance of vitamin A to brain function, behavior and learning. Mol Nutr Food Res. 2010;54(4):489-495.

31. Lane JT. The role of retinoids in the induction of nerve growth factor: a potential treatment for diabetic neuropathy. Transl Res. 2014;164(3):193-195.

32. Scalabrino G, Mutti E, Veber D, et al. Increased spinal cord NGF levels in rats with cobalamin (vitamin B12) deficiency. Neurosci Lett. 2006;396(2):153-158
Neuropsychiatric Disease and Treatment

\section{Publish your work in this journal}

Neuropsychiatric Disease and Treatment is an international, peerreviewed journal of clinical therapeutics and pharmacology focusing on concise rapid reporting of clinical or pre-clinical studies on a range of neuropsychiatric and neurological disorders. This journa is indexed on PubMed Central, the 'PsycINFO' database and CAS,

\section{Dovepress}

and is the official journal of The International Neuropsychiatric Association (INA). The manuscript management system is completely online and includes a very quick and fair peer-review system, which is all easy to use. Visit http://www.dovepress.com/testimonials.php to read real quotes from published authors. 